Kentaro Kasuga, Iwanori Murakami, Masaki Ono and Yoshinori Ando

\title{
Development of traveling wave propulsion mechanism with a permanent magnet vibration
} motor

ABSTRACT. Currently, autonomous underwater vehicles use screw propellers as the propulsion mechanism. However, screw propellers generate air bubbles that cause environmental changes and affect sound measurements around the probes. In addition, screw propellers can damage aquatic life. Therefore, we developed a propulsion mechanism using a traveling wave that imitates the movement of a fish. This device consists of a vibrating motor that uses a permanent magnet. This can reduce power consumption and generate high thrust. We produced a propulsion mechanism that uses a traveling wave and evaluated its basic structure and characteristics through an experiment.

Keywords: permanent magnet, traveling wave, vibration motor

Nanotechnology Perceptions 15 (2019) 220-226

doi: 10.4024/N24KA19A.ntp.15.03 